\title{
Der Spitalrat im Wandel der Zeit: von der grauen Eminenz zum aktiven Coach
}

\author{
Spitalräte sind die Verwaltungsräte der Spitäler. Sie überprüfen die unternehmeri- \\ schen Ziele im Rahmen der finanziellen Mittel und sorgen für ein gesundes Mass an \\ Kritik gegenüber der Geschäftsleitung. Die Anforderungen an den Spitalrat sind mit \\ Einführung der DRGs gestiegen. Mangelnde zeitliche Verfügbarkeit und fehlende \\ fachliche Kompetenz vermindern die Effizienz eines Spitalrats.
}

\author{
Michael Zaugga, \\ Roland Müller ${ }^{b}$ \\ a Prof. Dr. med., MBA, \\ University of Alberta \\ b Prof. Dr. iur., \\ Rechtsanwalt und Notar, \\ Universität St. Gallen
}

Korrespondenz:

Prof. Dr. med. Michael Zaugg, MBA

Dept. of Anesthesiology and Pain Medicine University of Alberta Clinical Sciences Building 113 St 83 Ave

CA-Edmonton, AB T6G 2G3

michael.zaugg[at]ualberta.ca
Good boards are pretty uncomfortable places and that's where they should be. (Sir Christopher Hogg)

Spitalräte sind die Verwaltungsräte der Spitäler. Bisher haben sie mehrheitlich das Dasein grauer Eminenzen geführt. Unter dem Druck der neuen wettbewerbsorientierten Spitalfianzierung, der Versicherungen, Patientenorganisationen und nicht zuletzt der Medien geraten sie aber immer mehr ins Rampenlicht der Öffentlichkeit. Zwei aktuelle Ereignisse seien hier kurz erwähnt, welche die zunehmende Bedeutung der Spitalräte illustrieren und gewisse Schwachstellen und Verbesserungsbedarf aufzeigen. So liess sich die Vizepräsidentin eines Spitalverbundes mit über 100000 CHF pro Jahr entschädigen, was zu breiter Kritik in den Medien führte. Unter anderem wurden an einem einzigen Tag gleich drei Sitzungspauschalen in Rechnung gestellt und damit begründet, dass der Verwaltungsrat operativ tätig werden musste, um Defizite in der Führung zu beheben. Das andere Beispiel ist eine «Revolte» gegen einen langjährigen Spitaldirektor, der im Interesse des Spitalverbundes Abteilungen zusammenlegen und schliessen musste. Bei dieser Geschichte führten Intransparenz, politisches Gezänke und fehlende strategische Weitsicht des Spitalrats zur Entlassung des Spitaldirektors und in der Folge zu einem «Leadership-Gau». Der folgende Artikel befasst sich mit der Frage, was die Funktionen des Spitalrates sind und wie die Arbeit im Spitalrat zum Nutzen der Spitäler optimiert werden kann.

\section{Welche Aufgaben hat ein Verwaltungsrat bzw. ein Spitalrat?}

Während die Geschäftsleitung (Team des Spitaldirektors bzw. CEO) für die täglichen operativen Geschäfte zuständig ist, ist der Verwaltungsrat bzw. Spitalrat für die erfolgreiche Ausrichtung und strategische Führung des Spitals verantwortlich. Er definiert die Langzeitstrategie und Ziele des Unternehmens, erstellt Reglemente, etabliert Ausschüsse (Auditkomitee,

\section{Les conseils d'administration} au fil du temps: de l'éminence grise au coaching actif

Le présent article cherche à déterminer comment optimiser le travail des conseils d'administration pour que celui-ci profite mieux aux hôpitaux. La tâche principale de ces conseils d'administration consiste en premier lieu à évaluer continuellement les objectifs entrepreneuriaux à l'aune des moyens financiers disponibles et à faire preuve d'esprit critique envers la direction des établissements. Le nouveau financement hospitalier en vigueur depuis 2012 a fondamentalement changé l'environnement stratégique des hôpitaux. D'éventuelles lacunes au sein des conseils d'administration peuvent avoir un impact sur la planification stratégique, la gestion des risques et dans l'interaction avec la direction de l'hôpital. Pour améliorer la gouvernance dans les hôpitaux suisses, les conseils d'administration doivent réunir davantage de médecins et ne pas servir uniquement de «cumul des mandats». Leurs membres doivent pratiquer la recherche active d'informations et disposer d'un code d'éthique en matière de gestion. Les conseils d'administration doivent jouer un rôle proactif lors du développement et de l'implémentation de standards de qualité et de sécurité. Par ailleurs, le temps des mandats délivrés à vie devrait être révolu. Enfin, il faut tenir compte du fait que le manque de temps couplé à des compétences spécifiques insuffisantes donne souvent lieu à des plaintes et à des actions en responsabilité pénale. 
Tabelle 1

Nach Obligationenrecht hat der einzelne Verwaltungsrat die hier aufgeführten Rechte und Pflichten.

\begin{tabular}{|c|c|}
\hline Rechte & Pflichten \\
\hline $\begin{array}{l}\text { Recht auf } \\
\text { Teilnahme an der } \\
\text { Generalversamm- } \\
\text { lung, auch wenn } \\
\text { ein VR nicht } \\
\text { Aktionär ist (und } \\
\text { seit dem 1.1.2008 } \\
\text { auch nicht sein } \\
\text { muss) }\end{array}$ & $\begin{array}{l}\text { Der Gesamtverwaltungsrat muss über eine ausreichende fachliche } \\
\text { Qualifikationen verfügen, um die undelegierbaren Aufgaben zu erfüllen: } \\
\text { - Oberleitung des Spitals inkl. Entwicklung und Umsetzung der } \\
\text { Unternehmensstrategie } \\
\text { - Sicherstellung eines ausgewogenen Verhältnisses von Zielen } \\
\text { und Mitteln inkl. Risiko Management } \\
\text { - Bestellung und Beaufsichtigung der Geschäftsleitung inkl. Erteilung } \\
\text { von notwendigen Weisungen und Festlegung der Entschädigungen } \\
\text { - Festlegen der Organisation inkl. Vorgabe der Zeichnungsberechtigung } \\
\text { und der Nachfolgeregelungen } \\
\text { - Ausgestaltung des Rechnungswesens und der Finanzkontrolle } \\
\text { - Erstellung des Geschäftsberichts } \\
\text { - Beachtung von Gesetz, Statuten und Reglementen (Compliance) }\end{array}$ \\
\hline
\end{tabular}

Recht auf Treuepflicht, doch kann ein Verwaltungsrat u.U. in konkurrierenden

Information, Firmen tätig sein, ohne seine Treuepflicht zu verletzen.

Einsicht und Zutritt

(gemäss Gesetz

aber nur über den

VR-Präsidenten)

Recht auf

Entschädigung*

Ausstandspflicht: Ein Verwaltungsrat muss in den Ausstand treten (keine Statements mehr abgeben und muss den Sitzungsraum verlassen), wenn seine persönlichen Interessen nicht mehr mit dem Spital übereinstimmen.

Recht auf Man-

datsniederlegung

* Das Gesetz spricht von angemessener Entschädigung (die meisten Entschädigungen liegen heute unter 45000 CHF pro Jahr, bei grossen und börsenkotierten Unternehmen sind diese meist über 100000 CHF pro Jahr. Ob Grund-, Pauschal/Spesen- oder Erfolgshonorar oder Gewinnbeteiligung wird nicht weiter spezifiziert. Nach den Autoren scheint eine Kombination von fixer Entschädigung und

Spesenabgeltung am sinnvollsten zu sein. Im Kanton Bern (regionale Spitalzentren) gelten folgende Honorierungen (September 2005): VR-Präsident 24500 CHF fix pro Jahr und VR-Mitglied 10500 CHF fix pro Jahr. Vom Kanton übernommen werden zudem die Prämien einer Organhaftpflicht-

versicherung zum Schutze der Verwaltungsräte.

Kompensationskomitee, Nominationskomitee), wählt, überwacht, evaluiert und (falls nötig) entlässt die Mitglieder der Geschäftsleitung. Er ist bei privatrechtlichen Aktiengesellschaften für die Antragstellung und die Auszahlung von Dividenden zuständig und kann den Aktionären Kapitalerhöhungen durch Ausgabe neuer Aktien vorschlagen (Privatkliniken wie z.B. Medi-Clinic Corporation Ltd.). Dabei ist eine klare Eignerstrategie mit versorgungspolitischen, finanzpolitischen und personalpolitischen Zielen sowie mit Vorgaben für Allianzen, Partnerschaften, regionale Verankerung, Zusammensetzung des Spitalrates, Finanzkontrolle, Revision und Berichterstattung von zentraler Bedeutung. Die Rechte und Pflichten des einzelnen Verwaltungsrates, aber auch des VRGremiums sind vom Gesetzgeber im Obligationenrecht relativ klar, aber nicht abschliessend definiert worden. In Tabelle 1 sind sie summarisch zusammengefasst [1]. Die wesentlichen Aufgaben des Gesamtverwaltungsrates sind demnach erstens das kontinuierliche Überprüfen der unternehmerischen Ziele mit den vorhandenen finanziellen Mitteln und zweitens ein gesundes Mass an Kritik gegenüber der Geschäftsleitung. Die im Obligationenrecht aufgelisteten Rechte und Pflichten, die grundsätzlich nur für

Spitäler, die als privatrechtliche Aktiengesellschaften organisiert sind, Gültigkeit haben, können für die Spitalräte anderer Organisationsformen analog herangezogen werden. Bei öffentlichen Spitälern ist hingegen primär das öffentliche Recht massgebend. Dort hat der Spitalrat insbesondere auch die rechtsstaatlichen Grundsätze der Verhältnismässigkeit und der schonenden Rechtsausübung zu beachten.

\section{Drastische Veränderungen der Rahmen- bedingungen für die Corporate Governance im DRG-Zeitalter}

Mit der neuen Spitalfinanzierung seit 2012 hat sich das strategische Umfeld in der schweizerischen Spitallandschaft grundlegend geändert [2]. Die öffentlichen Spitäler konkurrieren mit den privaten Anbietern. Leistungsaufträge werden kompetitiv und basierend auf Qualitätsdaten vergeben. Die Konkurrenz zwischen den öffentlichen Spitälern selbst hat ebenfalls zugenommen («Wettrüsten»). Es kommt im Rahmen der vertikalen und horizontalen Integration zu Netzwerkbildungen und Allianzen. Die Versicherer, die Politik und die Patienten stellen immer höhere Ansprüche.

Die rasanten und z.T. schnelllebigen Entwicklungen in der Medizin sowie der zunehmende Mangel an Fachkräften und Spezialisten sind weitere grosse Herausforderungen. Mit diesen Veränderungen kommen auch vermehrt Veränderungen auf dem Gebiete des Gesundheitspolizeirechts zu tragen (z. B. Datenschutz). Hinzu kommt die mit der Revision des Krankenversicherungsgesetzes eingeführte «freie Spitalwahl». Um diesen neuen Bedingungen gerecht $\mathrm{zu}$ werden, braucht es eine angepasste Corporate Governance in den Spitälern, insbesondere auch auf Ebene Spitalrat. Klare Eigentumsverhältnisse sind zwingend, damit keine Wettbewerbsverzerrung entsteht. So haben beispielsweise öffentliche Spitäler, deren Trägerschaft nicht Eigentümerin der Spitalliegenschaften ist - sondern z. B. der Kanton - gegenüber Spitälern, deren Trägerschaft gleichzeitig auch Eigentümerin der Liegenschaften ist, massive Wettbewerbsnachteile, da beispielsweise bauliche Massnahmen sehr aufwendig mit der Eigentümerin koordiniert werden müssen und allenfalls auch noch dem obligatorischen Finanzreferendum unterstehen.

\section{Welche Spitalräte sind am «effizientesten»? - Erfahrungen aus der Literatur}

In der Fachwelt haben sich einige Faktoren herauskristallisiert, die eng mit der Effizienz eines Verwaltungsrates/Spitalrates korrelieren. Nicht ganz überraschend scheint, dass weniger, aber dafür gut vorbereitete Verwaltungsratssitzungen (ca. 6 pro Jahr) von grösserem Nutzen sind. Der Spitalrat sollte auch aktives Leadership Mentoring betreiben mit dem Ziel, falls nötig innerhalb nützlicher Frist, einen geeigneten Nachfolger für den Spitaldirektor (CEO) zu finden. Zudem scheint es für einen Spitalrat nicht von 
Vorteil zu sein, den CEO als Mitglied im Spitalrat zu haben (er kann beratend zu den Sitzungen beigezogen werden). Auf jeden Fall sollte der Spitalratspräsident nicht auch die CEO-Position innehaben («Selbstbeaufsichtigung»). Profit und Ranking sind besser in Spitälern, wo keine Mandate auf Lebzeiten vergeben werden bzw. die Verwaltungsratsmandate zeitlich klar limitiert sind und wo ein eigenes Finanzkomitee des Spitalrates als Kontrollorgan die externe Auditierung zusätzlich ergänzt [3]. Die Grösse des Spitalrates hängt von der Strategie und lokalen Gegebenheiten ab und korreliert nicht mit der Profitabilität oder Effizienz. Jedoch zeigen mehrere Studien, dass ärztliche Expertise im Spitalrat klar die Profitabilität eines Spitals verbessern kann. Dies ist auch im Einklang mit Beobachtungen, wonach die medizinische Bildung des CEO eines Spitals (Direktor) mit einem besseren Ranking des Spitals einhergeht $[4,5]$. US Studien zeigen, dass im Mittel nur 25\% der Spitalräte auch einen ärztlichen Hintergrund haben.

Eine Studie aus Deutschland identifizierte die Präsenz von Ärzten im Spitalrat als der entscheidende Faktor für einen besseren ROA («return on as-

\section{«Bei 10 zufällig ausgewählten grösseren Schweizer Spitälern lag der Anteil von Ärzten im Spitalrat zwischen 0-20\%.»}

set»), während die Präsenz von Politikern mit einer reduzierten Profitabilität einhergeht [6]. Insbesondere sog. «inside physicians» (Mediziner aus dem eigenen Spital aber ohne Wahlrecht im Verwaltungsrat) sollen einen Vorteil darstellen und zu besseren Margen beitragen.

Weitere wichtige Faktoren für die Effizienz eines Spitalrates sind nach der Literatur auch der Wille zur kontinuierlichen Weiterbildung, die aktive Informationsbeschaffung aus dem Betrieb, der Wille, kritischen (oft auch unangenehmen) Fragen nachzugehen und schliesslich auch der Wille, die nötige Zeit in das Mandat zu investieren. Je höher die Anzahl der Verwaltungsratsmandate der einzelnen VR-Mitglieder ist, desto grösser ist auch die Gefahr, dass die benötigte Zeit für das Mandat nicht mehr zur Verfügung steht, denn viel Zeit muss neben der eigentlichen Sitzungszeit auch in das Studium des Geschäftsverlaufes und der Finanzrapporte investiert werden. Es wird davon ausgegangen, dass ein anspruchsvolles Mandat mindestens 1-2 Arbeitstage pro Monat beansprucht. Effiziente Verwaltungsräte haben keinen Platz für passive «Ämtlisammler». Als prominentes Beispiel eines «Ämtlisammlers» wurde der CEO der Lufthansa, der neben seinem neuen Verwaltungsratsmandat als Verwaltungsratspräsident bei Roche auch Verwaltungsratsmandate bei
Stadler Rail, Swiss, Iatra, Avenir Suisse etc. innehat, in den Medien publik gemacht.

Im Rahmen der Umsetzung der Abzockerinitiative musste der Verwaltungsrat von Roche kürzlich die maximale Anzahl Mandate ihrer Topmanager festlegen. Roche hat die Limite bei 15 Mandaten angesetzt (davon höchstens 5 börsenkotierte Unternehmen). Dies wird aber von vielen Fachleuten als zu hoch eingestuft, denn die nötige Zeit zur seriösen Erledigung der vielen Mandate ist fast unmöglich aufzubringen. Coca-Cola Enterprises, Inc. scheint da restriktiver zu sein und verlangt, dass bei gleichzeitiger CEO-Tätigkeit höchstens zwei weitere Mandate erlaubt sind [7]. Es ist zu bedenken, dass mangelnde zeitliche Verfügbarkeit zusammen mit fehlender fachlicher Kompetenz im Hinblick auf Buchhaltung, Finanzen und Fachwissen oft Ausgang von Verantwortlichkeitsklagen sind, denn Nichtwissen entlastet den Verwaltungsrat nicht. Der Verwaltungsrat kann grundsätzlich zivilrechtlich (z.B. Schadenersatzklage), straftrechtlich (z. B. Veruntreuung) oder administrativrechtlich (Entzug der Berufsberechtigung) belangt werden. Versichern kann er sich jedoch nur für das zivilrechtliche Risiko (Berufshaftpflichtversicherung oder Organhaftpflichtversicherung).

\section{Was sind die Anforderungen an einen Spitalrat von heute?}

Die Finanzkrise und nun auch die rasanten Veränderungen im Gesundheitswesen haben klargemacht, wie wichtig die Aufgaben der Verwaltungsräte/ Spitalräte sind [8]. Defizite können insbesondere im strategischen Planen, Risikomanagement und im Bereiche der Führung der Geschäftsleitung (des CEO) geortet werden. Ein Spitalrat muss heute wie ein privater Unternehmer denken und als unabhängiger Insider proaktiv sich um die strategischen Prioritäten des Spitals kümmern. Es genügt nicht mehr, eine Strategie der «Noses in, fingers out!» zu betreiben. Finanziellen Engpässen, Betreibungen und Prozessen muss im Sinne eines Krisenmanagements unverzüglich nachgegangen werden und entsprechende Massnahmen (Stopp von Investitionen, Personalabbau, Kündigung von Verträgen, sofern von Relevanz evtl. Kapitalerhöhung und/oder Verzicht auf Dividendenauszahlungen) eingeleitet werden. In Krisensituationen nimmt das Finanzmanagement eine zentrale Rolle ein [1].

Um als Spitalrat Mehrwert zu generieren, ist ein proaktives Handeln und insbesondere ein kontinuierliches aktives Coaching des CEO (Spitaldirektors) vonnöten. Dazu braucht der einzelne Spitalrat aber ein klares praktisches Verständnis, wie ein Spital funktioniert und profunde Kenntnisse im Gesundheitswesen, gepaart mit Erfahrung aus der Geschäftswelt. Dies ist auch deshalb mehr und mehr nötig, weil der Spitalrat heute immer mehr Verantwortung im Bereich Quality and Safety Management übernehmen muss [9]. Ohne medizinisches Know-how 


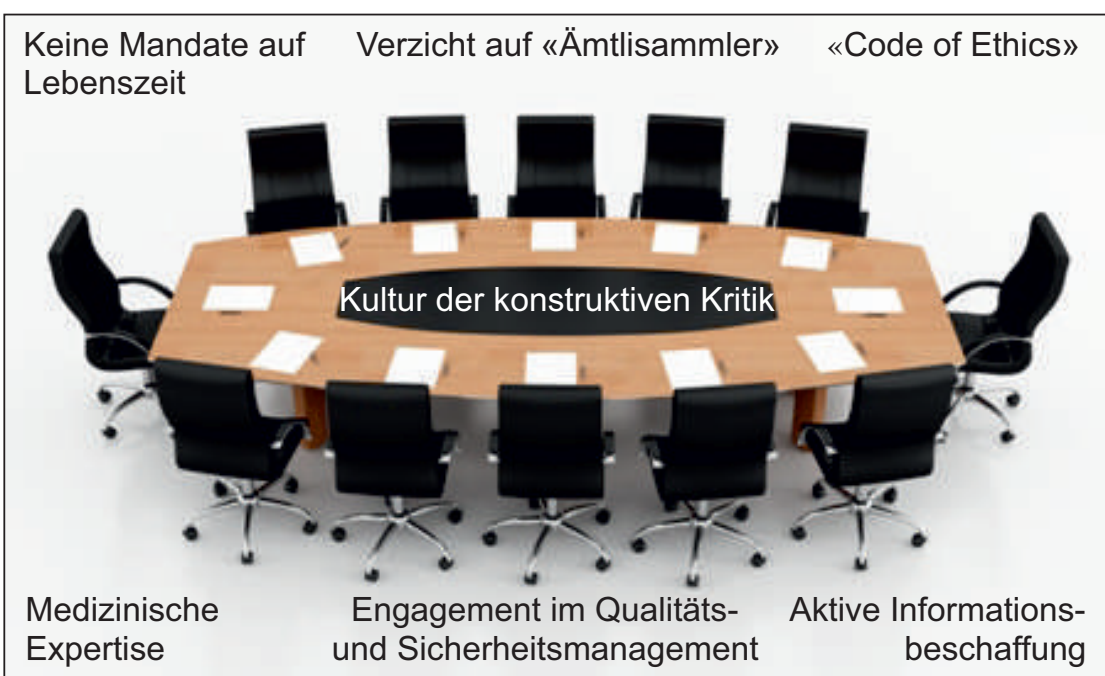

Abbildung 1

Welche Faktoren begünstigen Effizienz?

im Spitalrat muss für die Lösungsfindung bei solchen Problemen medizinisches Wissen teuer eingekauft werden.

\section{Überlegungen im schweizerischen Kontext}

Folgende Punkte sollten im Zusammenhang mit einer effizienten Corporate Governance auf Stufe Spitalrat überprüft werden:

- Es ist zu überdenken, ob im Zeitalter der zunehmenden Komplexität von Businessentscheidungen im Gesundheitswesen nicht mehr Spitalräte ein besser ausgewiesenes medizinisches Fachwissen haben sollten. Bei 10 zufällig ausgewählten grösseren Schweizer Spitälern lag der Anteil von Ärzten im Spitalrat zwischen 0-20\%. Nichtmedizinische Unterstützung, die nicht das Kerngeschäft betrifft (beispielsweise rechtliche Beratung und Vertretung) kann hingegen ohne weiteres ausgelagert werden.

- Das «Ämtlisammeln» scheint eine Untugend in Verwaltungsräten zu sein. Mehr Bescheidenheit und Transparenz bezüglich der Mandate mit Handelsregistereintrag wäre für öffentliche und teilprivatisierte Spitäler wünschenswert.

- Eine aktive Kultur der Informationsbeschaffung (sog. «Holschuld», nicht «Bringschuld») seitens des Spitalrats sollte auch an unseren Spitälern herrschen, setzt aber medizinische Expertise voraus. In den General Motors Company Board of Directors Corporate Governance Guidelines wird spezifisch festgehalten, dass Verwaltungsräte sich aktiv um Information aus ihrer Firma bemühen müssen und die Produktionsanlagen regelmässig besuchen sollten [10].

- Öffentliche, aber auch teilprivatisierte Spitäler sollten einen «Code of Ethics» für ihr Management erstellen, der im Sinne eines Sarbanes-
Oxley Act (vom US Senat verfügtes Gesetz zur Verbesserung der Verantwortlichkeit und Transparenz der Buchhaltung in Firmen als Antwort auf Skandale bei Enron und Tyco) Missbräuche verhindern kann.

- Nicht zuletzt sollten heutige Spitalräte auch eine proaktive Rolle bei der Entwicklung und Implementierung von nationalen und internationalen Qualität- und Sicherheitsstandards übernehmen, z.B. mit der Einführung von sog. «Board Quality Scorecards» [9]. Dies verlangt medizinisches Verständnis und Wissen und kann nur glaubhaft vertreten werden, wo dieses auch vorhanden ist.

Mit den in Abbildung 1 zusammengefassten Anpassungen kann der Spitalrat seinen unterschiedlichen Rollen als Supervisor, Vernetzer und immer mehr auch als Coach im DRG-Zeitalter besser gerecht werden.

\section{Literatur}

(Weitere Literatur bei den Autoren.)

1 Roland Müller, Staad. Rechtliche Anforderungen an den Spital-VR und die Spital-Leitung (www.advocat. ch); fundierte Details bei Müller, Lipp, Plüss. Der Verwaltungsrat. 4. Aufl. Zürich; 2014. (Mit einer umfangreichen Checkliste zu den VR-Aufgaben, S. 798-803)

2 Zaugg M, Gattiker A. SwissDRG ist der Katalysator für eine unternehmerische Ausrichtung. Competence. $5 / 2012$.

3 Culica D, Prezio E. Hospital board infrastructure and functions: the role of governance in financial performance. International Journal of Environmental and Public. Health. 2009;9:862-73.

4 Prybil LD. Size, composition, and culture of high-performing hospital boards. American Journal of Medical Quality. 2006;21:224-9.

5 Veronesi G, Kirkpatrick I, Vallascas F. Clinicians on the board: What difference does it make? Social Science \& Medicine. 2013;77:147-55.

6 Kuntz L, Pulm J, Wittland M. Hospital Governance and the structure of German hospital supervisory boards. Gesundheitswesen. 2013; Sep 30. Epub ahead of print.

7 Coca-Cola Enterprises. Inc. Board of directors guidelines on significant corporate governance issues. http://ir.cokecce.com/phoenix. zhtml?c=117435\&p=irol-govguidelines

8 Wong SCY. Elevating board performance: the significance of director mindset, operating context, and other behavioral and functional considerations. www.worldfinancialreview.com

9 Goeschel CA, Berenholtz SM, Culbertson RA, Jin L, Pronovost PJ. Board Quality Scorecards: measuring improvement. American Journal of Medical Quality. 2011;26:254-60.

10 General Motors Company Board of Directors Corporate Governance Guidelines. www.gm.com/ content/dam/gmcom/COMPANY/Investors/ Corporate Governance/PDFs/DCGC.pdf 